\title{
Taking care of medical students: the pillars of future healthcare
}

\author{
Sudhakar Selvaraj, ${ }^{1,2}$ (iD Vineeth John ${ }^{1}$ (D) \\ ${ }^{1}$ Faillace Department of Psychiatry and Behavioral Sciences, McGovern Medical School, The University of Texas Health Science Center at \\ Houston, Houston, TX, USA. ${ }^{2}$ UTHealth Student Counseling Services, McGovern Medical School, University of Texas Health Science Center, \\ Houston, TX, USA.
}

Very few occupations require training as long and arduous as the medical profession. Despite that, becoming a physician is a professional achievement that is often associated with societal trust and respect, a better lifestyle, and, more importantly, personal life satisfaction. Getting into medical school is a very competitive process worldwide, and it is just the beginning of the path to becoming a physician. The demands of medical school can be enormous, and maintaining high standards of education throughout the course is often especially stressful.

Stress during medical school is well recognized. Stressrelated symptoms and "burnout" are prevalent (52\%) and are associated with unprofessional conduct. ${ }^{1}$ A systematic review that extracted data from 167 cross-sectional studies worldwide estimated the prevalence of depression in medical students was $27 \%$, and the prevalence of suicidal ideation was $11.1 \%$ in a subsample of studies. ${ }^{2}$ Most importantly, only $15.7 \%$ of the medical students who screened positive for depression had sought psychiatric treatment. ${ }^{2}$ This suggests that a substantial number of students are untreated and suffering silently. Untreated depression and stress-related problems often lead to substance abuse, relationship problems, career dissatisfaction, and dropouts, as well as increase the risk of suicide. Studies show that physicians are also at higher risk of depression and suicide than the general population. ${ }^{3}$

Research suggests that the increased rate of depression in medical students is potentially due to a combination of personal, situational, and school-related factors. ${ }^{4}$ Personal history of psychological problems, maladaptive coping styles, lack of family and social support, and financial difficulties may all contribute to the risk of depression and stress-related illnesses. However, situational and structural factors such as a stressful learning environment, blame culture, bullying, lack of mentorship, increased stigma about mental illness, and limited availability of counseling programs and wellbeing initiatives in medical schools have also been cited for contributing to increased stress among medical students.

Correspondence: Sudhakar Selvaraj, Faillace Department of Psychiatry and Behavioral Sciences, McGovern Medical School, University of Texas Health Science Center, 1941 East Road, Houston, TX, USA.

E-mail: Sudhakar.selvaraj@uth.tmc.edu

Submitted Apr 15 2020, accepted Apr 28 2020, Epub Jun 122020.
Comprehensive assessment and targeted interventions are critical in reducing stress among students and thus preventing deterioration in long-term mental health. In this issue, Bermudez et al. ${ }^{5}$ have applied an adapted form of the Unified Protocol for Transdiagnostic Treatment of Emotional Disorders (UP) as a preventive intervention for depression in medical students $(n=62)$. They conducted an open-label, single-session, cognitive-behavioral intervention for medical students in small groups, followed up for 90 days. The treatment intervention focused on improving mindfulness, emotional awareness, and cognitive flexibility. The authors found a significant reduction in subjective depression symptoms and quality of life scores in the study participants who completed the program. Undoubtedly, the strengths of this study are the preventive approach and brief noninvasive nature of the intervention, which can be useful in medical schools in different environments. However, the study has a few notable limitations, which include the modest sample size, open-label design without a control group, and selection bias because a large number of students who had pre-existing psychological symptoms were included. Nevertheless, these preliminary findings are promising and warrant further exploration in a controlled trial to establish the efficacy of this therapy approach in medical students.

All medical students require easier access to counseling, mentoring, and support in order to address their most critical personal and psychological needs. The medical curriculum involves a substantial amount of study materials, exams, and evaluations, and all can increase anxiety and stress. Revisiting the medical school curriculum and the structure of student evaluations would make it less stressful for students while simultaneously retaining the necessary rigorousness of training standards. Awareness of mental health to tackle stigma, creating a supportive culture, and making student mentorship and peer-support more accessible are all necessary for improving the quality of medical students' wellbeing.

How to cite this article: Selvaraj S, John V. Taking care of medical students: the pillars of future healthcare. Braz $\mathrm{J}$ Psychiatry. 2021;43:4-5. http://dx.doi.org/10.1590/1516-4446-2020-1037 
The recent COVID-19 pandemic is showing the world the exceptional dedication to service of all frontline healthcare professionals (including physicians) in saving patients, often with minimal personal protective equipment, thereby putting their lives at risk both acutely and in the long term. In these unprecedented circumstances, the essentiality of healthcare professionals to save lives seems more clear than ever. Governments, healthcare leadership, and educational institutions should invest more in the wellbeing and mental health of medical students, physicians, and healthcare professionals. Taking care of the wellbeing of medical students - future frontline physicians and essential pillars of our society - is the need of the hour.

\section{Disclosure}

The authors report no conflicts of interest.

\section{References}

1 Dyrbye LN, Massie FS Jr, Eacker A, Harper W, Power D, Durning $\mathrm{SJ}$, et al. Relationship between burnout and professional conduct and attitudes among US medical students. JAMA. 2010;304: 1173-80.

2 Rotenstein LS, Ramos MA, Torre M, Segal JB, Peluso MJ, Guille C, et al. Prevalence of depression, depressive symptoms, and suicidal ideation among medical students: a systematic review and metaanalysis. JAMA. 2016;316:2214-36.

3 Panagioti M, Geraghty K, Johnson J, Zhou A, Panagopoulou E, Chew-Graham $C$, et al. Association between physician burnout and patient safety, professionalism, and patient satisfaction: a systematic review and meta-analysis. JAMA Intern Med. 2018;178:1317-30.

4 Dyrbye LN, Thomas MR, Shanafelt TD. Medical student distress: causes, consequences, and proposed solutions. Mayo Clin Proc. 2005;80:1613-22.

5 Bermudez MB, Costanzi M, Macedo MJ, Tatton-Ramos T, Xavier AC, Ferrão YA, et al. Improved quality of life and reduced depressive symptoms in medical students after a single-session intervention. Braz J Psychiatry. 2020;42:145-52. 